\title{
MAGNETICALLY MODULATED MICROWAVE ABSORPTION (MMMA) AT LOW MAGNETIC FIELDS IN (BEDT - TTF) - SUPERCONDUCTORS §
}

\author{
\#P. Bele, \#H. Brunner, "D. Schweitzer and ${ }^{+} H . J$. Keller \\ \# Max Planck Institut für Medizinische Forschung, AG Molekülkristalle, Jahnstr.29, \\ 69120 Heidelberg, Germany \\ * 3. Physikalisches Institut der Universität Stuttgart, Pfaffenwaldring 57, \\ 70550 Stuttgart, Germany \\ + Anorganisch Chemisches Institut der Universität Heidelberg, \\ Im Neuenheimer Feld 270, 69120 Heidelberg, Germany
}

( received 20. May 1994 by J. Kuhl)

(Accepted 1 July 1994)

\begin{abstract}
We report magnetically modulated microwave absorption ( MMMA) at low magnetic fields in crystals of the organic superconductors $\alpha_{t}$-(BEDT-TTF $\left.)_{2}\right]_{3}$ and $\mathrm{k}$-(BEDT-TTF $)_{2} \mathrm{Cu}(\mathrm{NCS})_{2}$. in both cases a strong MMMA - signal can easily be found below the critical temperature $T_{C}$. In some selected crystals series of narrow, periodically spaced lines are observed comparable to corresponding spectra of the CuO - type high $\mathrm{T}_{\mathrm{C}}$ superconductors. A model developed for the HTSC 's single crystals is used to interpret the results of the organic superconductors.
\end{abstract}

\section{Keywords}

A. Superconductors

D. Fluxpinning and creep

\section{Introduction}

In the last few years magneticaily modulated microwave absorption at low magnetic fields has been extensively studied in HTSC 's. MMMA is a very efficient and sensitive method for investigating superconductivity of a sample. Of great interest are measurements on single crystals where highly resolved periodically structured spectra in weak external magnetic fields are observed. Their dependence on the orientation of the crystal, the temperature, field modulation and microwave threshold power is still a challenge for a comprehensive theory of the MMMA - effect / 1-6/.

For organic superconductors based on bis(ethylenedithio)tetrathiafulvalene (BEDT-TTF) only a few measurements showing the appearance of the MMMA - effect were reported so far, but no narrow, periodically spaced lines were observed / 7 91 .

In this letter we present experiments of magnetically modulated microwave absorption at low magnetic fields in crystals of the organic super- conductors $\alpha_{t}-(B E D T-T T F)_{2} l_{3}$ and $x$-(BEDTTTF) ${ }_{2} \mathrm{Cu}(\mathrm{NCS})_{2}$.

In both cases we observed spectra showing a temperature and field dependence of the MMMA signal below the critical temperature $T_{C}$ and for the first time we are able to present experimental results which exhibit a pattern of highly resolved lines periodic to the applied external magnetic field $\mathbf{B}_{\mathbf{0}}$. These high resolution was found only for crystals of very good quality.

The experimental results are interpreted with the theory developed for the HTSC 's / 2, 10, 11 /.

\section{Experimental}

$\alpha$-(BEDT-TTF $)_{2} l_{3}$ crystals were grown from a THF - solution by electrochemical methods $/ 12 /$. By tempering the crystals for 4 to 6 days at a temperature of $75^{\circ} \mathrm{C}$ a structural transition to the $\alpha_{t}$ - phase occurs which has been investigated with several techniques / 13, $14 /$. Due to the stress

$\$$ Reported at the "Frahjahrstagung Festkorperphysik der Deutschen Physikalischen

Gesellschaft, Münster 1994 "; ISSN 0420 - 0195, p.1634 
during the $\alpha$ - to the $\alpha_{t}$ - phase transformation the crystals showed a strong mosaicity seen in visible polarized reflected light, but the crystal structure of $\alpha_{t}$ (BEDT-TTF) $2 / 3$ could not be determined. However, the reflections which are observed in Weissenberg - pictures for $\alpha_{t}$ (BEDT-TTF $)\left._{2}\right|_{3}$ are almost the same as for $\beta$-(BEDT-TTF $)_{2} l_{3}$. That means the unit cells of $\alpha_{4}$ (BEDT-TTF $)_{2} l_{3}$ and $\beta$ (BEDT-TTF) ${ }_{2} / 3$ are very similar $/ 15 l$. The tempered $\alpha_{t}$ - modification is stable at room temperature and the crystals exhibit a superconducting transition near $8 \mathrm{~K} / 13 /$.

$\mathrm{K}$-(BEDT-TTF $)_{2} \mathrm{Cu}(\mathrm{NCS})_{2}$ is an organic superconductor with one of the highest transition temperature $\left(T_{C} \approx 10.4 \mathrm{~K}\right) / 16 /$. The crystals were grown by electrochemical methods as described earlier in $/ 17$ I.

The magnetically modulated microwave absorption measurements were carried out with a VARIAN E line EPR spectrometer which operates in the $X$ band, and an Oxford instruments ESR910 helium cryostat. The plate - like $\alpha_{-}-(B E D T-T T F)_{2} / 3$ or the distorted - hexagon - shaped K-(BEDT$\pi \mathrm{TF})_{2} \mathrm{Cu}(\mathrm{NCS})_{2}$ crystals were fixed with silicon grease to the flat end of a suprasil quartz holder in a $\mathrm{TE}_{102}$ microwave cavity. All samples were oriented with the microwave magnetic field $\mathbf{B}_{\mathbf{m w}}$ perpendicular and the external magnetic field $\mathbf{B}_{0}$ parallel to the conducting plane of the crystal. $\mathbf{B}_{\mathbf{0}}$ was modulated with $100 \mathrm{kHz}$. The measurements of the highly resolved spectra were carried out at a temperature of $1.8 \mathrm{~K}$.

\section{Results}

As mentioned before MMMA is a good method for detecting the superconducting properties of a sample.

Fig. 1 shows the typical MMMA - signal as a function of the temperature of the two organic superconductors $\alpha_{\mathrm{t}}$-(BEDT-TTF) ${ }_{2}{ }_{3}$ and $k$-(BEDT$T \mathrm{TF})_{2} \mathrm{Cu}(\mathrm{NCS})_{2}$ measured with a high modulation amplitude. Such signals allow the determination of the onset temperature $T_{c}$ of superconductivity. One obtains for $\alpha_{t}$-(BEDT-TTF $)_{2} l_{3} T_{C} \approx 8 \mathrm{~K}$ and for $\mathrm{k}$-(BEDT-TTF $)_{2} \mathrm{Cu}(\mathrm{NCS})_{2} \mathrm{~T}_{\mathrm{C}}=10.4 \mathrm{~K}$. These results are in good agreement with the values known from conductivity, thermopower and Meissner - effect measurements.

Fig. 2 presents a MMMA - signal recorded with a high modulation amplitude as a function of the field $B_{0}$ in the range from $-5 \mathrm{mT}$ to $+3 \mathrm{mT}$ at a temperature of $1.8 \mathrm{~K}$ for a $\alpha_{4}$-(BEDT-TTF) ${ }_{2} / 3$ crystal. One can see an asymmetry of the signal which depends on the direction of the scan of $\mathbf{B}_{\mathbf{0}}$; a very small hysteresis of the MMMA - signal can be observed.

In figure 3 magnetically modulated microwave absorption spectra at low magnetic fields are shown for an $\alpha_{t}$-(BEDT-TTF) ${ }_{2} I_{3}$ crystal. The spectra exhibit a pattern of well resolved periodical lines as it is
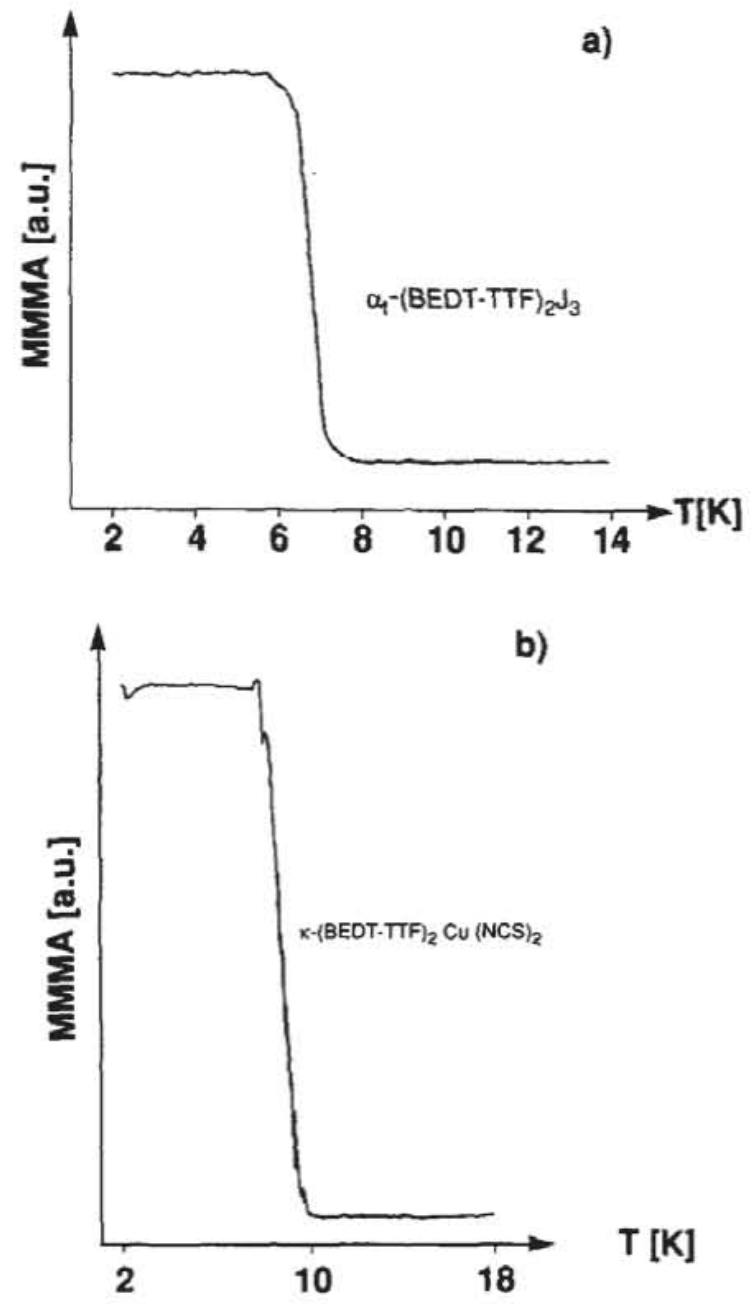

Fig. 1 MMMA - signal at low magnetic field with high modulation amplitude as a function of temperature for two samples of organic superconductors. a) $\alpha_{t}-(\text { BEDT-TTF })_{2} / 3$ and $\left.b\right) \kappa-$ (BEDT-TTF) 2 Cu(NCS) 2 . Microwave power is $\mathrm{P}_{\mathrm{mw}}$ $=2 \mathrm{~mW}$, modulation amplitude $3.2 \mathrm{mT}$ and $\mathrm{B}_{\mathrm{o}}=+1$ $\mathrm{mT}$.

obtained in YBaCuO single crystals / 1 - $6 /$. We can detect this line pattern over a very broad scan range of $B_{0}$. The range is as large as $10 \mathrm{mT}$ for the best crystal orientation. To receive the best line pattem it was necessary to adjust the measurement setup ( microwave power, modulation amplitude, crystal orientation etc. ) individually for each crystal. We note that the resolution of the periodical lines is very sensitive to the microwave power, in this case $80 \mu \mathrm{W}$. No signal could be detected below this threshold value. Another important feature is that the direction of the microwave field $\mathbf{B}_{\mathrm{mw}}$ must be perpendicular to the conducting plane of the crystal and to $\mathbf{B}_{\mathbf{o}}$. The average spacing between two lines is $\Delta \mathrm{B}_{\mathrm{O}} \approx 55 \mu \mathrm{T}$ and the equidlstant lines are observed over a total scan range of $10 \mathrm{mT}$ for this particular $\alpha_{t}$-(BEDT-TTF) ${ }_{2} l_{3}$ crystal. Fig. 3a) shows the MMMA - signal for a part ( $6 \mathrm{mT}$ ) of the total field range. In figure $3 b$ ) only a small range of the spectrum ( $1.6 \mathrm{mT}$ ) is presented for better 


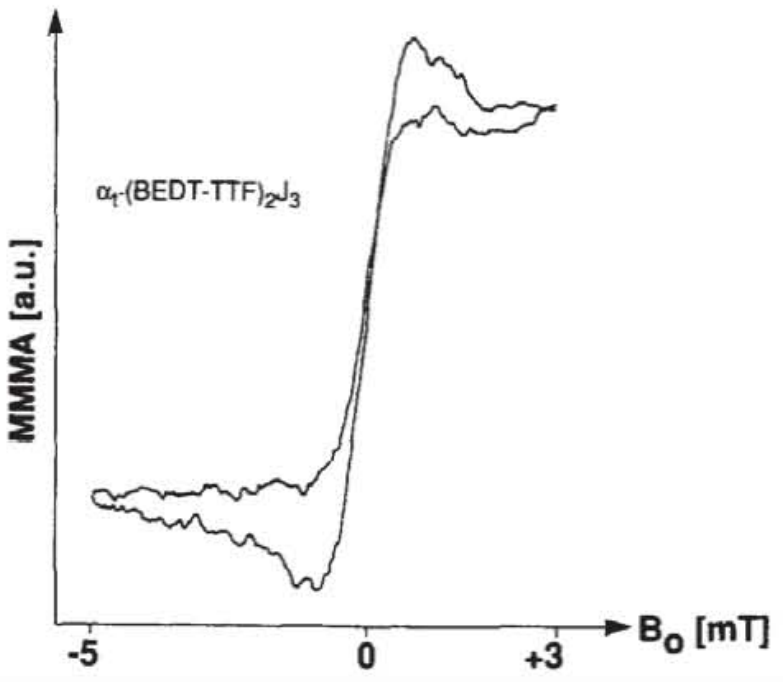

Fig. 2 Hysteresis of the MMMA - signal with high modulation amplitude at low magnetic field for a sample of $\alpha_{t}-(B E D T-T T F)_{2} l_{3}$. Temperature is $1.8 \mathrm{~K}$, microwave power $P_{m w}=2 \mathrm{~mW}$, modulation amplitude $3.2 \mathrm{mT}$ and the cycled range of the external magnetic field $B_{0}$ varies from $-5 \mathrm{mT}$ to $+3 \mathrm{mT}$.

displaying the shape and the spacing of the lines. One can clearly see that every single line consists of two separate peaks, i. e. an upper and a lower lobe, as predicted from the theory $/ 10 /$.

The MMMA - signal of the other organic superconductor, $\mathrm{k}$-(BEDT-TTF $)_{2} \mathrm{Cu}(\mathrm{NCS})_{2}$, is shown in figure 4. As for the $\alpha_{t}$-crystal the degree of resolution of the line pattern of the signal depends sensitively
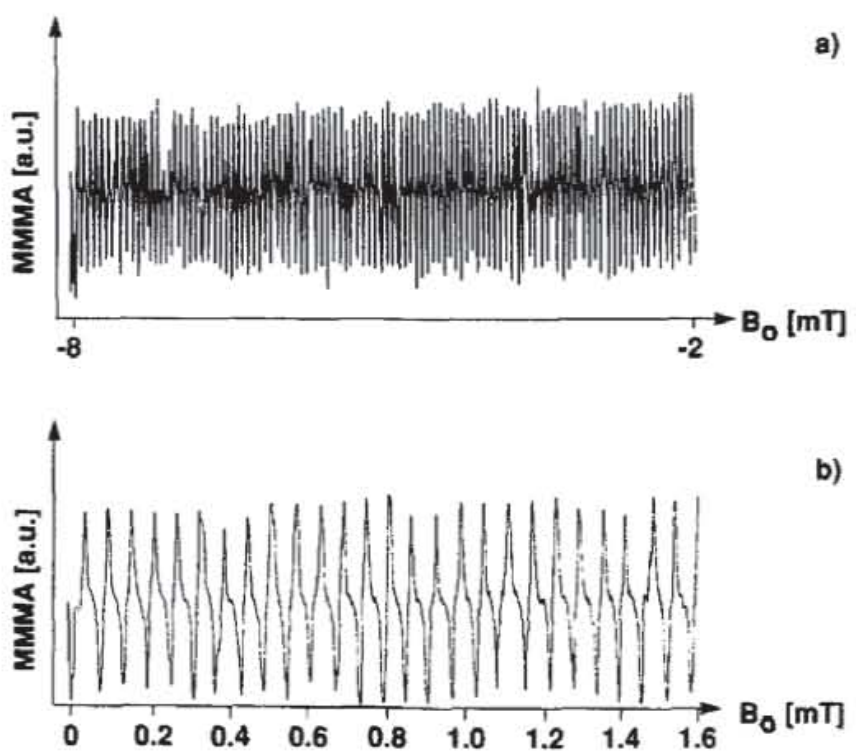

Fig. 3 Low field MMMA as a function of the external magnetic field $\mathbf{B}_{0}$ for $\alpha_{t}$-(BEDT-TTF) ${ }_{2} \mathrm{l}_{3}$. Temperature is $1.8 \mathrm{~K}, P_{\text {mw }}=0.08 \mathrm{~mW}$ and modulation amplitude $10 \mathrm{mT}$.( Both spectra shown are only parts of the whole spectrum for a better display of the resolved lines).

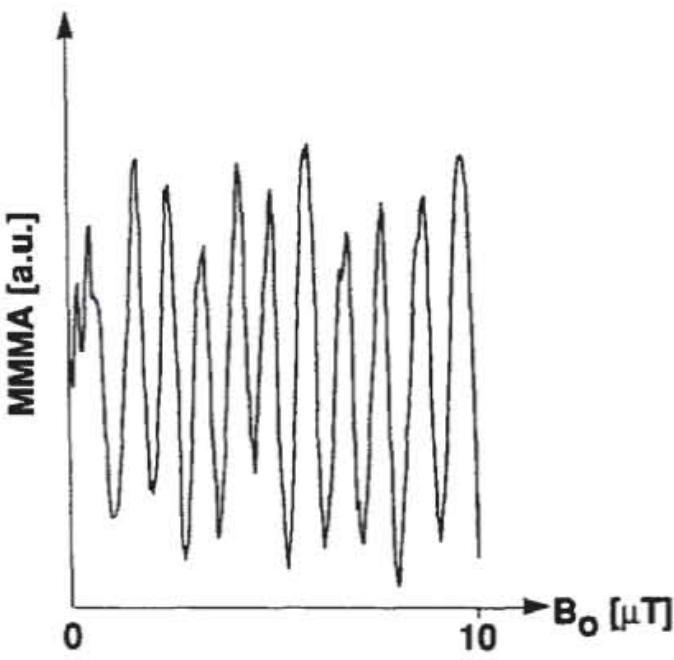

Fig. 4 Low field MMMA as a function of the external magnetic field $B_{0}$ for $\kappa$-(BEDT-TTF $)_{2} \mathrm{Cu}(\mathrm{NCS})_{2}$. Temperature is $1.8 \mathrm{~K}$, modulation amplitude $10 \mathrm{mT}$ and $P_{m w}=0.008 \mathrm{~mW}$. Direct detection with a second field modulation of $38 \mathrm{~Hz}$ and an amplitude of $100 \mathrm{mT}$ via storage oscilloscope.

on the parameter setup. The most important difference in the MMMA - signal of this organic superconductor and that of $\alpha_{\mathrm{t}}$-(BEDT-TTF) ${ }_{2} \mathrm{l}_{3}$ is the much smaller spacing $\Delta B_{0}=0.9 \mu \mathrm{T}$ of the lines. To get the best resolution we had to go to a microwave power $P_{m w}=8 \mu \mathrm{W}$ which was only slightly above the threshold value. This forced us to detect this spectrum without AFC - control. Under this condition the stability of the spectrometer was not sufficient for a longtime field scan. Therefore we detected the MMMA - spectrum with a second field modulation of $38 \mathrm{~Hz}$ and an amplitude of $100 \mathrm{mT}$ directly on a storage oscilloscope. By varying $\mathbf{B}_{0}$ a highly resolved line pattern could be observed over a total field range of $5 \mathrm{mT}$ with this technique.

\section{Theory and discussion}

An interpretation of the magnetically modulated microwave absorption spectra showing a series of equidistant lines is possible with a theoretical model first described by A.H. Silver and J.E. Zimmerman / $10 /$ and modified for HTCS 's by T. Xia and D. Stroud / $11 /$ and H. Vichery, F. Beuneu and P. Lejay $/ 21$.

In the model one considers a superconducting planar loop with an area $\mathrm{A}$ containing $\mathrm{N}$ identical Josephson junctions. Under the influence of an external magnetic field $B_{0}$ the loop supports a screening supercurrent and - as usual for a type II superconductor - the loop is penetrated by a magnetic flux $\Phi_{\text {ext }}$ which is an integer multiple of the flux quantum $\Phi_{0}$. The magnetic energy of the loop shows a periodic structure with broad minima depending on the flux density $\Phi_{\text {ext }} / \Phi_{\mathrm{O}}$. The energy of an individual state of the loop is characterized by 
an integer parameter $p$, which is connected to the flux through the loop. In the regime of the maxima where the supercurrent in the loop approaches the critical current in the Josephson junctions, the energies of two states $p$ and $p+1$ overlap. An essential assumption of the model is that only under the condition of a critical current a transition between two overlapping energy states and a change of the flux through the loop is possible. The observed microwave absorption is then understood as losses in the shunt resistance of the Josephson junction, when the junction becomes normal conducting.

The quantitative analysis of this model given by the above mentioned authors predicts that a spectrum of periodic line pairs (lobe up and down ) is to be expected if $\mathbf{B}_{0}$ is scanned and that every single line is due to the increase or decrease of the flux in the loop by one flux quantum. From the spacing $\Delta \mathrm{B}_{\mathrm{O}}$ between two lines the size of the area $A$ of the loop can be estimated as

$$
A=\Phi_{0} / \Delta B_{0}
$$

where the flux quantum $\Phi_{0}$ is given to $2.07 \times$ $10^{-15} \mathrm{~T} / \mathrm{m}^{2}$

Inserting the measured values of $\Delta B_{0}$ into this equation one obtains

$A_{1} \approx 3.8 \times 10^{-11} \mathrm{~m}^{2} \approx 38 \mu \mathrm{m}^{2}$ for $\alpha_{\mathrm{t}}$ (BEDT-TTF $)_{2} \mathrm{I}_{3}$ and

$A_{2} \approx 2.3 \times 10^{-9} \mathrm{~m}^{2} \approx 2300 \mu \mathrm{m}^{2}$ for $\mathrm{k}$-(BEDTTTF $)_{2} \mathrm{Cu}(\mathrm{NCS})_{2}$.

The large difference in the size of these areas ( their ratio is $\approx 60$ ) can be explained by the fact that the surface of $\alpha_{t}$-(BEDT-TTF $)_{2} / 3$ crystals possesses a mosaic structure after the tempering process. This leads to a lot of surface defects which evidently limit the areas of the loops. The high resolution in the MMMA - spectra of the crystals of $k$-(BEDTTTF $)_{2} \mathrm{Cu}(\mathrm{NCS})_{2}$ was reproducible only with selected crystal specimens. An average crystal of this organic superconductor shows a spectrum with a few broad lines which are relatively independent of the microwave power $P_{m w}$. The existance of a threshold of the microwave power $P_{m w}$ can be explained by the Josephson junction model, too.

When we compare the size of the areas $A_{1}$ and $A_{2}$ inferred from our measurements of the organic superconductors with analog values for HTSC 's / $18-20 /$ we find that $A_{1}$ of $\alpha_{t}$-(BEDT-TTF) $)\left._{2}\right|_{3}$ is $\sim 2$ times smailer than the smallest HTSC value while $\mathrm{A}_{2}$ of $\mathrm{k}$-(BEDT-TTF $)_{2} \mathrm{Cu}(\mathrm{NCS})_{2}$ is $\sim 2$ times larger than the largest value found for HTSC 's. The reason for the larger disparity of the organic superconductors compared to the HTSC's may be connected with the larger difference of the surface qualities in the organic supeconductors. Nervertheless the real nature of the Josephson junctions and of the crystal defects limiting the loop areas is still unclear.

\section{References}

/1/ KW. Blazey, H.M. Portis, K.A. Müller, F.H. Holtzberg

Europhys. Lett. 6, 457 (1988)

12/ H. Vichery, F. Beuneu, P. Lejay Pysica C 159, 823 (1989)

13/ A. Dulcic, R.H. Crepeau, H.J. Freed Physica C 160, 223 (1989)

14 K.W. Blazey, H.M. Portis, F.H. Holtzberg Physica C 157, 16 (1989)

15/ K. Kish, S. Tyagi, C. Krafft Phys. Rev. B 44, 225 (1991)

16/ M. Puri, R. Durny, L. Kevan Physica C 190, 210 (1992)

I7I M. Thakur, R.C. Haddon, S.H. Glarum Journal of crystal growth 106, 724 (1990)

18/ A.A. Zakhidov, A. Ugawa, K. Yakushi, K. Imaeda, H. Inokuchi, I.I. Khairullin, P.K. Khabibullaev Physica C 185 - 189, 2669 (1991)

19/ R.C. Haddon, S.H. Glarum, S.V. Chichester, A.D. Ramirez, N.M. Zimmerman Phys. Rev. B 43, 2642 (1991)
110/ A.H. Silver, J.E. Zimmerman Phys. Rev. 157, 317 (1989)

/11/ T. Xia, D. Stroud Phys. Rev. B 39, 4792 (1989)

/12/ K. Bender, I. Hennig, D. Schweitzer, K. Dietz, H. Endres, H.J. Keller

Mol. Cryst. Liq. Cryst. 108, 359 (1984)

113/ D. Schweitzer, P. Bele, H. Brunner, E. Gogu,

U. Haeberlen, I. Hennig, T. Klutz,

R. Zwietlik, H.J. Keller

Z. Phys. B - Condensed Matter B 67, 489 (1987)

/14/ S. Kahlich, S. Gărtner, D. Schweitzer, H.J. Keller

Synth. Met. 42, 2019 (1991)

/15/ G.O. Baram, L.I. Buravov, L.C. Degtariev, M.E. Kozlov, V.N. Laukhin,

E.E. Laukhina, V.G. Onishenko, V.I. Pokhodnia, M.K. Shenikman, R.D. Shibaeva, E.B. Yagubskii JETP Lett. 44, 489 (1987) 
/16/ Urayama, H. Yamochi, G. Saito, K. Nozawa, T. Sugano, M. Kinoshita, S. Sato,

K. Oshima, A. Kawamoto, J. Tanaka

J. Chem. Lett. 55 (1988)

/17/ S. Gärtner, E. Gogu, I. Heinen, H.J. Keller, T. Klutz, D. Schweitzer

Sol. State Comm. 65, 1531 (1988)

/18/ D. Hoffmeister, O. Dobbert, K.P. Dinse,

W. Goldacker, T. Wolf

Europhys. Lett. 8, 369 (1989)
$119 / \mathrm{H}$. Vichery, F. Rullier-Albenque, F. Beuneu, P. Lejay Physica C 162 - 164, 1583 (1989)

120/ A. Pöppl, L. Kevan, H. Kimura, R.N. Schwartz Phys. Rev. B 46, 8559 (1992) 